\title{
Advanced systems in public transport
}

\author{
Marie Schmidt ${ }^{1} \cdot$ Stefan $\operatorname{Vo}^{2}{ }^{(D)}$
}

Accepted: 22 May 2017/Published online: 14 June 2017

(C) Springer-Verlag Berlin Heidelberg 2017

This special issue of Public Transport: Planning and Operations assembles selected papers from the 'Conference on Advanced Systems in Public Transport' (CASPT) in Rotterdam, the Netherlands, in July 2015.

Like CASPT, this issue covers a broad range of topics in public transportation, including strategical, tactical, and operational planning, real time control and reoptimization, as well as simulation of public transportation systems.

The mission of CASPT is to bring together and to initiate and strengthen collaborations among academic researchers and practitioners in the area of public transportation. This is reflected in the special issue: conceptual and methodological innovations are complemented with contributions in software which bridges the gap between theory and practice. What joins the papers is the common will to improve the state-of-the-art in public transportation.

The order of contributions in this special issue follows the usual order of planning steps in public transportation planning, followed by contributions on real-time control and reoptimization, and concludes with two-very different-simulation studies and an approach to characterize a public transportation system by OD-pair mode choice.

The first two papers are concerned with line planning. The first paper addresses an often neglected step in strategical public transportation planning. Many

Marie Schmidt

schmidt2@rsm.nl

Stefan Voß

stefan.voss@uni-hamburg.de

1 Department of Technology and Operations Management, RSM-Rotterdam School of Management, Erasmus University Rotterdam, PO Box 1738, 3000 DR Rotterdam, The Netherlands

2 Institute of Information Systems, University of Hamburg, Von-Melle-Park 5, 20146 Hamburg, Germany 
optimization formulations for railway line planning take a set of possible lines as input. Gattermann, Schiewe, Harbering, and Schöbel address the question on how to generate such a line pool. In experiments on the German high-speed railway network, they show that the choice of the line pool is crucial for both operational costs and number of direct OD-connections. In the line planning model by Perea, De-Los-Santos, Laporte, and Mesa, frequencies are assigned to the lines from a line pool with the objective of maximizing net profit. Hereby, passengers' mode choice is taken into account using a logit model. In this way, higher frequencies, on the one hand, lead to lower costs, but on the other hand, they reduce passenger waiting times and thus increase the demand captured, leading to higher revenues.

Public transportation systems suffer frequently from delays and disturbances. Thus, there is a need for robust public transportation systems. Trap, Huisman, and Goverde propose and evaluate three different line plans for winter conditions in the Netherlands with respect to controllability and capacity shortage. Even under regular weather conditions, small delays are unavoidable. Hence, timetables should be able to absorb a certain amount of delay. Maróti proposes a branch-and-bound algorithm for an earlier stochastic programming formulation from literature which reduces the computation time for finding a robust modification of a given reference timetable from several days to a few minutes on the Dutch railway network. Also Bärmann, Martin, and Schneider consider the effect of slight changes on a given timetable. Their goal, however, is not robustness against delays, but energyefficiency. They study and compare the problem under different objective functions which can aim to decrease maximum average power consumption or fluctuations in power consumption. Experiments on the timetables of railway stations in several German cities show that significant reductions are possible.

Borndörfer, Hoppmann, and Karbstein study timetabling from a passengeroriented perspective. They provide theoretical examples to show that different ways of modeling passengers' route choice can lead to very different timetables, and show that this is, to smaller extent, also applicable to real-world instances in a case study of the public transportation system of the city of Wuppertal in Germany.

After a timetable is determined, rolling stock has to be assigned to the trips in the timetable, taking into account the operational costs as well as demand. Lin, Barrena, and Kwan propose a model which incorporates two levels of demand: a minimum level that needs to be fulfilled, and a desired level, which enters the objective function together with operational costs. They test their model on the Central Scotland railway network.

A new challenge arises in the scheduling of electric buses: due to the limited capacity of batteries, these need to be recharged during the day. Van Kooten Niekerk, Hoogeveen, and van den Akker propose several models for this problem which differ in the degree of detail in which they model the recharging process and test these on data from Leuven in Belgium.

The difficulty in rostering and scheduling of (public transportation) personnel arises from the multitude of regulations and legal constraints on the one hand, and objectives which are difficult to quantify, like employee satisfaction or safety, on the other hand. Weider, Schulz, Seidl, and Borndörfer aim at improving bus driver satisfaction, by integrating duty scheduling and rostering. They make use of so- 
called duty templates to couple the two problems and solve it using Benders' decomposition. Results on two large urban transportation networks are presented. Meanwhile, Snijders and Saldanha consider the job scheduling problem for security crews. They consider the objectives to minimize fare evasion as well as aggression against train personnel or passengers. The developed heuristic is built into a prototype to support decisions at the Netherlands Railways.

'Regular' public transportation offers can be combined with transportation on demand, to make a public transportation more inclusive. Posada, Andersson, and Häll develop two integer programming models for the dial-a-ride problem with timetabled fixed route service and compare these on a small problem instance. Schönberger does not include scheduled services into the planning, but allows transfers between different vehicles. A genetic algorithm is developed which provides good results on small benchmark instances, but is also applicable to large problem instances.

Even in the best planned public transportation systems, not everything goes (exactly) as planned, and adjustments need to be made. Advances in communication technology and computation speed provide the opportunity to support decision making in real time with the use of information technology. Lentink, Middelkop, and De Vries study the benefit of providing updated driving instructions to train drivers in a simulation study. They show that large benefits could be achieved already by adjusting driving speed in delay situations, even if the order of trains on tracks is not changed.

Sánchez-Martínez, Wilson, and Koutsopoulos go so far as to argue that highfrequency public transportation could be operated schedule-free, that is, the determination of timetables, rolling stock, and crew schedules could be replaced by real-time control and optimization. They propose to include only rolling stock and crew entry and exit times into operational planning, and assign these to duties in real time, allowing for constant adjustment of timetables and schedules. The problem is formalized, but due to its complexity and the very limited amount of time available for computations in real-time optimization, a simple heuristic is proposed. Tested on instances based on a single line, where running time was still small enough for realtime operations, the schedule-free approach turned out to be competitive with respect to a schedule-based approach, and even outperforms it on instances with delay.

Following the state-of-the-art schedule-based approach to public transportation planning, schedules have to be adjusted after delays and disruptions. Rückert, Lemnian, Blendinger, Rechner, and Müller-Hannemann develop a tool to support train dispatchers. Based on real-time information on delays and passenger flows, they evaluate wait-depart decisions by simulating future passenger flows. In extensive case studies on the German railway network, they show that following the recommendations from the dispatching tool, passenger delays can be reduced significantly. Verhaegh, Fioole, Huisman, and Vera Lizcano develop a fast heuristic for real-time crew rescheduling for small disruptions. In a first step, tasks that cannot be executed by the assigned driver anymore are recognized and removed from the drivers' duty. In a second step, the tasks are assigned to other drivers' duties. Hereby, the heuristic follows a smart enumeration strategy which allows to 
repair a feasible crew schedule in a few seconds for $73 \%$ of the considered instances.

Ghaemi, Cats, and Goverde classify rescheduling actions in case of major disruptions into three phases. They give an overview of the current rescheduling practice for major disruptions in the Netherlands and contrast it to disruption management approaches from the literature. After discussing applicability and limitations of these models, in particular with respect to rescheduling phases covered and degree of infrastructure detail, they illustrate an earlier method on an example from the Dutch railway network. Reuther, Borndörfer, Schlechte, and Grimm study the problem of re-optimizing a rolling stock schedule, with particular focus on reproducing as many key aspects of the reference schedule as possible. This is modelled by the use of what the authors call connection and rotation templates in a hypergraph model. Although the introduction of the rotation templates leads to a drastic reduction of computation times, these are still in the range of hours, making the model suitable for re-optimization for predictable disturbances of normal operations like, e.g., construction works, rather than real-time applications.

Optimization methods help to design/shape components of the public transportation networks having one (or sometimes: several) objective(s) in mind.

Simulation can be used to evaluate the impact of public transportation decisions with respect to a broad range of concepts. Ingvardson, Jensen, and Nielsen develop a mesoscopic simulation model to evaluate reliability of on-street public transit modes. Public transit vehicles are modelled microscopically, whereas other traffic is simulated macroscopically. The approach is demonstrated on a busy bus line in Copenhagen, Denmark.

Srikukenthiran and Shalaby develop a platform, which allows to link simulators for surface transit, rail transit, and movement of passengers in stations. Passengers are determined by an external routing module, and then they are modelled as agents and can move between the different simulators controlled by a coordination server. In a case study on the public transportation system of Toronto, the authors prove that the platform called Nexus can handle large scale real-world multi-modal transit networks.

A very different approach to evaluate (existing) public transportation systems is taken by Viggiano, Koutsopoulos, Wilson, and Attanucci who develop a method to characterize public transport systems by the mode choice of the passengers. They use the method to describe the model choice in the London public transportation system, distinguishing between OD-pairs traveling mostly by rail, bus, or a combination of both, based on data from the Oyster smartcard.

Finally, Ranjbari, Chiu, and Hickman investigate the potential of a new transportation mode, intercity high speed buses, on the Tucson-Phoenix corridor in Arizona, USA. Based on a stated preference survey, they compare passengers' mode choice, taking into account mode characteristics like travel time, frequency, and cost, but also characteristics of the passenger like gender and age, and of the trip, e.g., availability of public transport at origin and destination station.

Our special thanks go to the reviewers whose repeated and constructive feedback contributed greatly to the quality of the manuscripts in this issue. 